\title{
Solution Structure of a $\beta$-Peptide Ligand for hDM2
}

\author{
Joshua A. Kritzer ${ }^{\dagger}$, Michael E. Hodsdon§, and Alanna Schepartz ${ }^{\star}, \dagger, \ddagger$ \\ Departments of Chemistry and Molecular, Cellular and Developmental Biology, Yale University, \\ New Haven, Connecticut 06510, and Department of Laboratory Medicine, Yale School of \\ Medicine, New Haven, Connecticut 06520
}

\begin{abstract}
Recently, we described a $\beta$-peptide foldamer, $\boldsymbol{\beta 5 3 - 1}$ (Figure 1A), that assembles into a 14helix in aqueous solution, binds the oncoprotein $\mathrm{hDM} 2$ with submicromolar affinity, and inhibits the interaction of $\mathrm{hDM} 2$ with a peptide derived from the activation domain of $\mathrm{p} 53$ (p53AD). ${ }^{1}$ The intact recognition epitope of $\boldsymbol{\beta 5 3 - 1}$, including a high degree of helical structure, is required for selective inhibition of the p53AD-hDM2 interaction. Here, we present the solution structure of $\boldsymbol{\beta 5 3 - 1}$ in methanol. The structure reveals details of a helixstabilizing salt bridge on one helical face, novel "wedge into cleft" packing along another, and how distortions in the $\boldsymbol{\beta 5 3 - 1}$ 14-helix maximize presentation of the p53AD recognition epitope. These details deepen our understanding of how $\beta^{3}$-peptides fold and how they can be designed to form higher order structures and bind macromolecules. ${ }^{2,3}$
\end{abstract}

Two-dimensional NMR spectroscopy was performed using $5 \mathrm{mM} \boldsymbol{\beta 5 3 - 1}$ in $\mathrm{CD}_{3} \mathrm{OH}$ at 10 ${ }^{\circ} \mathrm{C}$. Previous circular dichroism and analytical ultracentrifugation experiments ${ }^{1}$ and the NMR line widths observed herein are consistent with a monomeric, 14-helical structure for ק53-1 under these conditions. The proton resonances of $\boldsymbol{\beta 5 3 - 1}$ were assigned unambiguously using TOCSY and natural abundance ${ }^{1} \mathrm{H}-1{ }^{13} \mathrm{C}$ HSQC spectra. ${ }^{4}$ ROESY experiments were then performed using mixing times of 200, 350, and $500 \mathrm{~ms}^{5}$ The observed series of $\mathrm{NH}-\mathrm{C}_{\alpha} \mathrm{H}$ ROEs confirmed the sequential assignment by providing a backbone "ROE walk". Three classes of medium-range ROEs characterize a 14-helical conformation: those between $\mathrm{H}_{\mathrm{N}}(i)$ and $\mathrm{H}_{\beta}(i+2), \mathrm{H}_{\mathrm{N}}(i)$ and $\mathrm{H}_{\beta}(i+3)$, and $\mathrm{H}_{\alpha}(i)$ and $\mathrm{H}_{\beta}(i+3)$. 6,7 All 20 potential medium-range interactions of this type were observed in the ROESY spectra of $\boldsymbol{\beta 5 3}-\mathbf{1}$; in addition, 27 additional medium-range ROEs between side chains three positions apart were also observed. ${ }^{4}$ The large number of medium-range ROEs observed by NMR provides clear evidence for a high level of 14-helix structure in $\boldsymbol{\beta 5 3}-1$; 449 ROEs quantified using a $350 \mathrm{~ms}$ mixing time were subsequently assigned and integrated using SPARKY. ${ }^{8}$ Peak volumes were converted to 151 upper-limit distance constraints ${ }^{4}$ and used to perform simulated annealing torsional dynamics on 100 random starting configurations of B53-1 using DYANA. ${ }^{4,9}$ No constraint violations were reported among the resulting 20 lowest-energy structures, which are shown in Figure 1B.

The ensemble of calculated structures of $\boldsymbol{\beta 5 3}-\mathbf{1}$ (Figure 1B) shows a 14-helix with an average backbone atom RMSD from the mean structure of $0.17 \pm 0.07 \AA$. The backbone torsions of individual structures deviate little from the mean, even at the termini (Figure 1C), illustrating the robustness of the $\boldsymbol{\beta 5 3 - 1}$ 14-helix in methanol. The helix is characterized by

\footnotetext{
alanna.schepartz@yale.edu.

$\dagger$ Department of Chemistry, Yale University.

\$Department of Molecular, Cellular and Developmental Biology, Yale University.

$\S$ Yale School of Medicine.
}

Supporting Information Available: Assignment tables, ROE-derived upper-distance limits. This material is available free of charge via the Internet at http://pubs.acs.org. 
approximately $1.61 \AA$ rise per residue and 3.0 residues per turn for residues $1-6$, with a slight unwinding to approximately $1.49 \AA$ rise per residue and 3.3 residues per turn for residues 7-10. This unwinding appears to be unique to $\boldsymbol{\beta 5 3}-\mathbf{1}$, as it was not observed in NMR structures of unrelated $\beta^{3}$-peptides with and without side chain ion pairing. ${ }^{6,7,10}$ Side chains are also well-defined among the lowest-energy structures, with an overall average heavy atom RMSD from the mean of $0.60 \pm 0.10 \AA$.

$\boldsymbol{\beta 5 3} \mathbf{- 1}$ contains four charged side chains arranged to favor formation of helix-stabilizing salt bridges on one 14-helix face. ${ }^{11}$ In all 20 low-energy structures, the terminal nitrogen of $\beta^{3} \mathrm{O} 7$ and the nearest terminal oxygen of $\beta^{3} \mathrm{E} 10$ are characterized by a consistent separation of $5.5 \pm 0.6 \AA$. The relative positions of the remaining two ion pairs fall into two subpopulations (Figure 2D). In 17 structures, the terminal nitrogen of $\beta^{3} \mathrm{O} 1$ and the nearest terminal oxygen of $\beta^{3} \mathrm{E} 4$ are closer $(5.4 \pm 0.9 \AA)$ than the equivalent atoms of $\beta^{3} \mathrm{E} 4$ and $\beta^{3} \mathrm{O} 7(6.8 \pm 0.9 \AA)$. By contrast, in the remaining three structures, the terminal nitrogen of $\beta^{3} \mathrm{O} 7$ and the nearest terminal oxygen of $\beta^{3} \mathrm{E} 4$ are closer $(3.6 \pm 0.4 \AA)$ than the equivalent atoms of $\beta^{3} \mathrm{O} 1$ and $\beta^{3} \mathrm{E} 4(7.7 \pm 1.3 \AA)$. This interplay among potential ion pairs suggests that the central salt bridge is weaker than those near the termini and supports the hypothesis that multiple interconnected ion pairs play a key helix-stabilizing role. ${ }^{11,12}$

Another feature incorporated into the design of $\boldsymbol{\beta 5 3}-\mathbf{1}$ was the inclusion of $\beta^{3}$-homovaline $\left(\beta^{3} \mathrm{~V}\right)$ residues at positions 2,5 , and 8 . It was long surmised ${ }^{11,13-15}$ and recently proven ${ }^{12}$ that $\beta^{3}$-amino acids branched at the first side chain carbon stabilize 14-helices, in stark contrast to the effects of such side chains on $\alpha$-helices. ${ }^{16}$ The $\boldsymbol{\beta 5 3}-\mathbf{1}$ structure provides a clear rationale for these observations. All 20 low-energy structures contain a unique arrangement of $\beta^{3}$-homovaline side chains in which one methyl group of a $\beta^{3} \mathrm{~V}$ side chain nestles into a cleft formed by the two methyl groups of another $\beta^{3} \mathrm{~V}$ side chain (Figure 2E). These interactions are especially noticeable between the side chains of $\beta^{3} \mathrm{~V} 5$ and $\beta^{3} \mathrm{~V} 8$, which are in VDW contact ${ }^{17}$ in 19 of 20 structures. Overall, interactions among the three $\beta^{3} \mathrm{~V}$ side chains bury $155 \pm 13 \AA^{2}$ of hydrophobic surface area from water $(24 \%$ of the surfaces of these side chains). These packing interactions may explain why these and other branched residues stabilize 14-helices ${ }^{12,18,19}$ and suggest new avenues for the design of 14helix bundles..$^{20,21}$

The remaining 14-helix face consists of residues that comprise the hDM2-binding epitope, namely, $\beta^{3}$-homoleucine $\left(\beta^{3} \mathrm{~L} 3\right), \beta^{3}$-homotryptophan $\left(\beta^{3} \mathrm{~W} 6\right)$, and $\beta^{3}$-homophenylalanine $\left(\beta^{3} \mathrm{~F} 9\right)$. We originally hypothesized that the side chains of these residues would form an extended hydrophobic surface that might mimic that of p53AD. ${ }^{1}$ Interestingly, the $\beta^{3} \mathrm{~F} 9$ side chain can access two specific conformations within the constraints used; the fact that this variability has been observed in another 14-helix structure ${ }^{7}$ implies that the side chain may indeed preferentially populate these rotamers within a 14-helix. The side chains of $\beta^{3} \mathrm{~W} 6$ and $\beta^{3} \mathrm{~L} 3$ are in VDW contact in all 20 structures, while the side chains of $\beta^{3} \mathrm{~W} 6$ and $\beta^{3}$-F9 are in VDW contact in the context of only one of $\beta^{3} \mathrm{F9}$ 's two preferred conformations (present in 6 of 20 low-energy structures). Overall, on average, the side chains of $\beta^{3} \mathrm{~L} 3$, $\beta^{3} \mathrm{~W} 6$, and $\beta^{3} \mathrm{~F} 9$ comprise a continuous, solvent-exposed hydrophobic surface area of 520 $\AA^{2}$. This value is comparable to the contact areas measured at the interfaces of transient homo- and heterodimeric protein complexes. ${ }^{22}$

As a consequence of the unexpected unwinding near the C-terminus of $\boldsymbol{\beta 5 3}-\mathbf{1}$, the $\beta^{3} \mathrm{~F} 9$ side chain is not aligned perfectly with the side chains of $\beta^{3} \mathrm{~L} 3$ and $\beta^{3} \mathrm{~W} 6$ along the helix axis (see Figure 1B). This subtle distortion may avoid steric repulsions between the large side chains of $\beta^{3} \mathrm{~F} 9$ and $\beta^{3} \mathrm{~W} 6$. In fact, it is unclear whether the unwinding near the $\mathrm{C}$-terminus, which is unique to $\boldsymbol{\beta 5 3 - 1}$, is due to more favorable ion pairing, more favorable $\beta^{3} \mathrm{~V}$ nesting interactions, or the need to avoid steric clashes on the recognition face containing large 
hydrophobic residues. As structures of other short, stable 14-helices are determined, it will be interesting to note what factors lead to similar distortions in the "ideal" 14-helix geometry.

Importantly, this subtle distortion allows the side chains comprising the $\boldsymbol{\beta 5 3}-\mathbf{1}$ recognition face to better mimic those on the p53AD $\alpha$-helix. Overlays between $\boldsymbol{\beta 5 3}-\mathbf{1}$ in an idealized 14-helical conformation and $\mathrm{p} 53 \mathrm{AD}$ bound to $\mathrm{hDM} 2^{23}$ revealed an imperfect alignment between the two ligands; while the $\beta^{3} \mathrm{~L} 3, \beta^{3} \mathrm{~W} 6$, and $\beta^{3} \mathrm{~F} 9$ side chains of $\boldsymbol{\beta 5 3}-\mathbf{1}$ could superimpose with their counterparts on $\mathrm{p} 53 \mathrm{AD}$, the 14-helix backbone could not completely fit within hDM2's binding groove. ${ }^{1}$ The comparable overlay with the solution structure of ק53-1 (Figure 2) shows no such conflict. In its solution conformation, $\boldsymbol{\beta 5 3}-\mathbf{1}$ can access all three of hDM2's hydrophobic pockets while occupying the same binding groove as $\mathrm{p} 53 \mathrm{AD}$ with no steric clashes. This fit demands subtle unwinding near the $\boldsymbol{\beta 5 3}-\mathbf{1}$ C-terminus that staggers the side chains, producing a $\beta^{3}$-peptide that is uniquely suited for $\alpha$-helix mimicry. Protein-protein interactions are notoriously difficult to inhibit with most ligand classes; ${ }^{24,25}$ the solution structure of $\boldsymbol{\beta 5 3}-\mathbf{1}$ suggests that $\beta$-peptide oligomers can present an extended, highly variable surface that could be used as a general platform to target these critical interfaces.

\section{Supplementary Material}

Refer to Web version on PubMed Central for supplementary material.

\section{Acknowledgments}

This work was supported by the NIH (GM 59843 to A.S. and AI 01806 to M.H.), the National Foundation for Cancer Research, and in part by a grant to Yale University, in support of A.S., from the Howard Hughes Medical Institute. J.A.K. is grateful to the NSF for a Predoctoral Fellowship.

\section{References}

1. Kritzer JA, Lear JD, Hodsdon ME, Schepartz A. J Am Chem Soc 2004;126:9468. [PubMed: 15291512]

2. Seebach D, Beck AK, Bierbaum DJ. Chem Biodiversity 2004;1:1111.

3. Cheng RP, Gellman SH, DeGrado WF. Chem Rev 2001;101:3219. [PubMed: 11710070]

4. Please see Supporting Information for details.

5. ROE intensities were linear in this range.

6. Arvidsson PI, Rueping M, Seebach D. Chem Commun 2001:649.

7. Etezady-Esfarjani T, Hilty C, Wuthrich K, Rueping M, Schreiber J, Seebach D. Helv Chim Acta 2002;85:1197.

8. Goddard, TD.; Kneller, DG. SPARKY 3. University of California; San Francisco, CA: 2004.

9. Guntert P, Mumenthaler C, Wuthrich K. J Mol Biol 1997;273:283. [PubMed: 9367762]

10. Rueping M, Mahajan YR, Jaun B, Seebach D. Chem-Eur J 2004;10:1607.

11. Hart SA, Bahadoor ABF, Matthews EE, Qiu XYJ, Schepartz A. J Am Chem Soc 2003;125:4022. [PubMed: 12670203]

12. Kritzer JA, Tirado-Rives J, Hart SA, Lear JD, Jorgensen WL, Schepartz A. J Am Chem Soc 2005;127:167. [PubMed: 15631466]

13. Raguse TL, Lai JR, Gellman SH. Helv Chim Acta 2002;85:4154.

14. Hamuro Y, Schneider JP, DeGrado WF. J Am Chem Soc 1999;121:12200.

15. Gung BW, Zou D, Stalcup AM, Cottrell CE. J Org Chem 1999;64:2176.

16. Chakrabartty A, Baldwin RL. Adv Protein Chem 1995;46:141. [PubMed: 7771317]

17. Creighton, TE. Proteins. W. H. Freeman and Co; New York: 1993.

18. Martinek TA, Fulop F. Eur J Biochem 2003;270:3657. [PubMed: 12950249] 
19. Glattli A, Seebach D, van Gunsteren WF. Helv Chim Acta 2004;87:2487.

20. Raguse TL, Lai JR, LePlae PR, Gellman SH. Org Lett 2001;3:3963. [PubMed: 11720580]

21. Cheng RP, DeGrado WF. J Am Chem Soc 2002;124:11564. [PubMed: 12296699]

22. Nooren IMA, Thornton JM. J Mol Biol 2003;325:991. [PubMed: 12527304]

23. Kussie PH, Gorina S, Marechal V, Elenbaas B, Moreau J, Levine AJ, Pavletich NP. Science 1996;274:948. [PubMed: 8875929]

24. Schepartz A, Kim PS. Curr Opin Chem Biol 1998;2:9. [PubMed: 9667922]

25. Sakurai K, Chung HS, Kahne D. J Am Chem Soc 2004;126:16288. [PubMed: 15600307] 
A

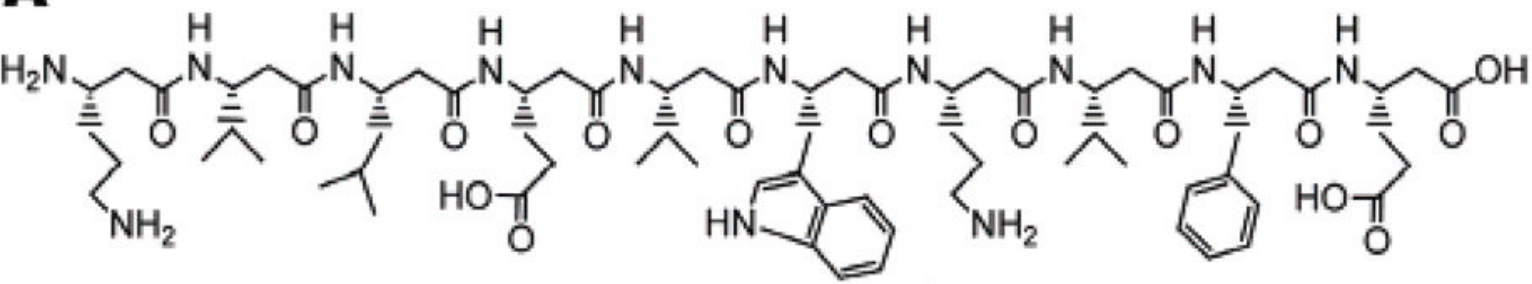

8
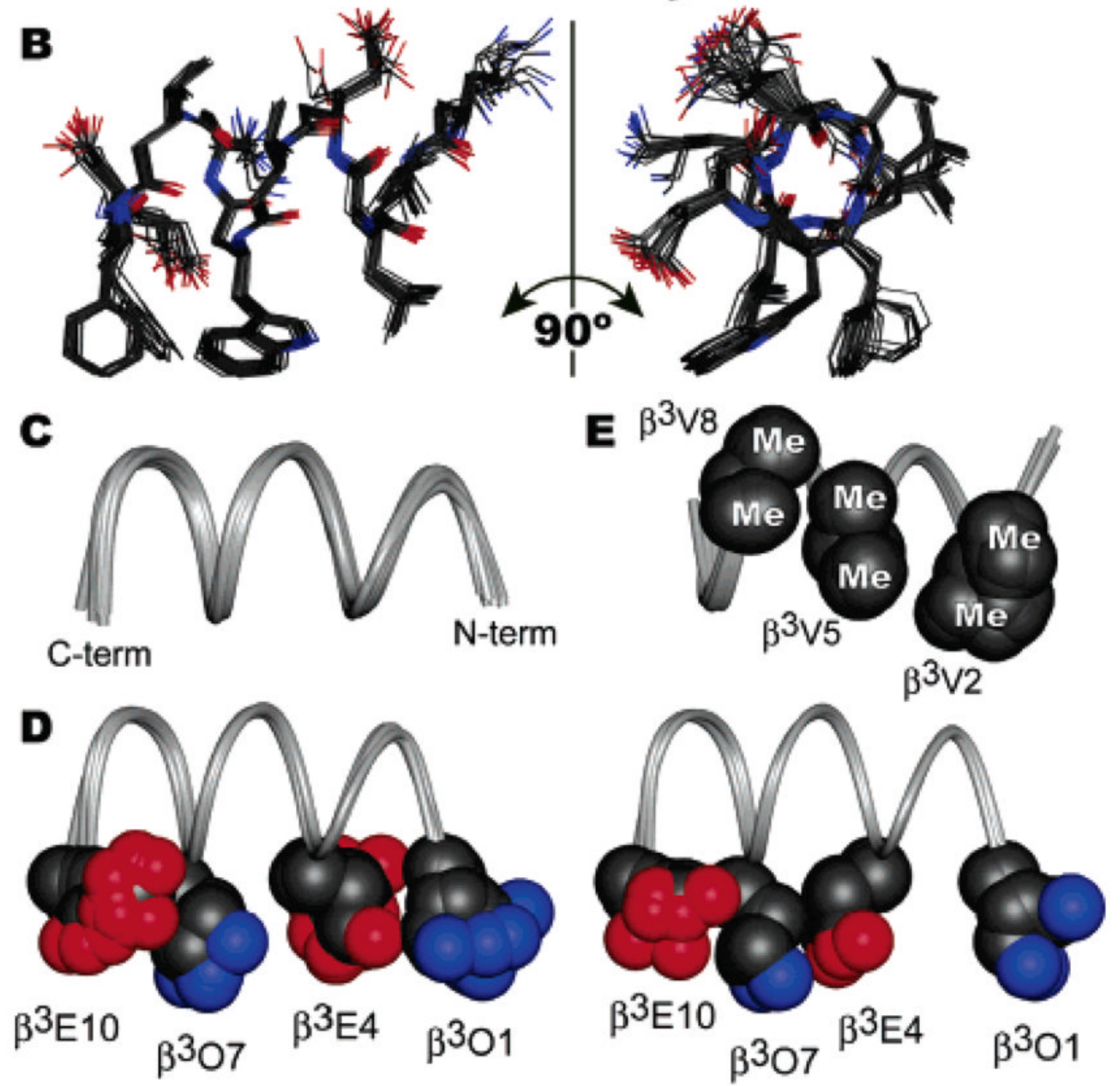

Figure 1.

(A) Chemical structure of $\boldsymbol{\beta 5 3}-\mathbf{1}$, shown with N-terminus at left. (B) Solution structure of 及53-1 in $\mathrm{CD}_{3} \mathrm{OH}$ at $10{ }^{\circ} \mathrm{C}$, shown as a bundle of 20 lowest-energy structures, with $\mathrm{C}$ terminus at left. (C) Ribbon representation of the backbones of 20 lowest-energy structures. (D) Two subpopulations of ion pairing configurations. Superposed at left are 17 structures in which $\beta^{3} \mathrm{O} 1$ and $\beta^{3} \mathrm{E} 4$ are proximal; superposed at right are three structures in which $\beta^{3} \mathrm{E} 4$ and $\beta^{3} \mathrm{O} 7$ are proximal. (E) Conformations of $\beta^{3}$-homovaline residues illustrating the "wedge into cleft" packing found in all 20 lowest-energy structures. 


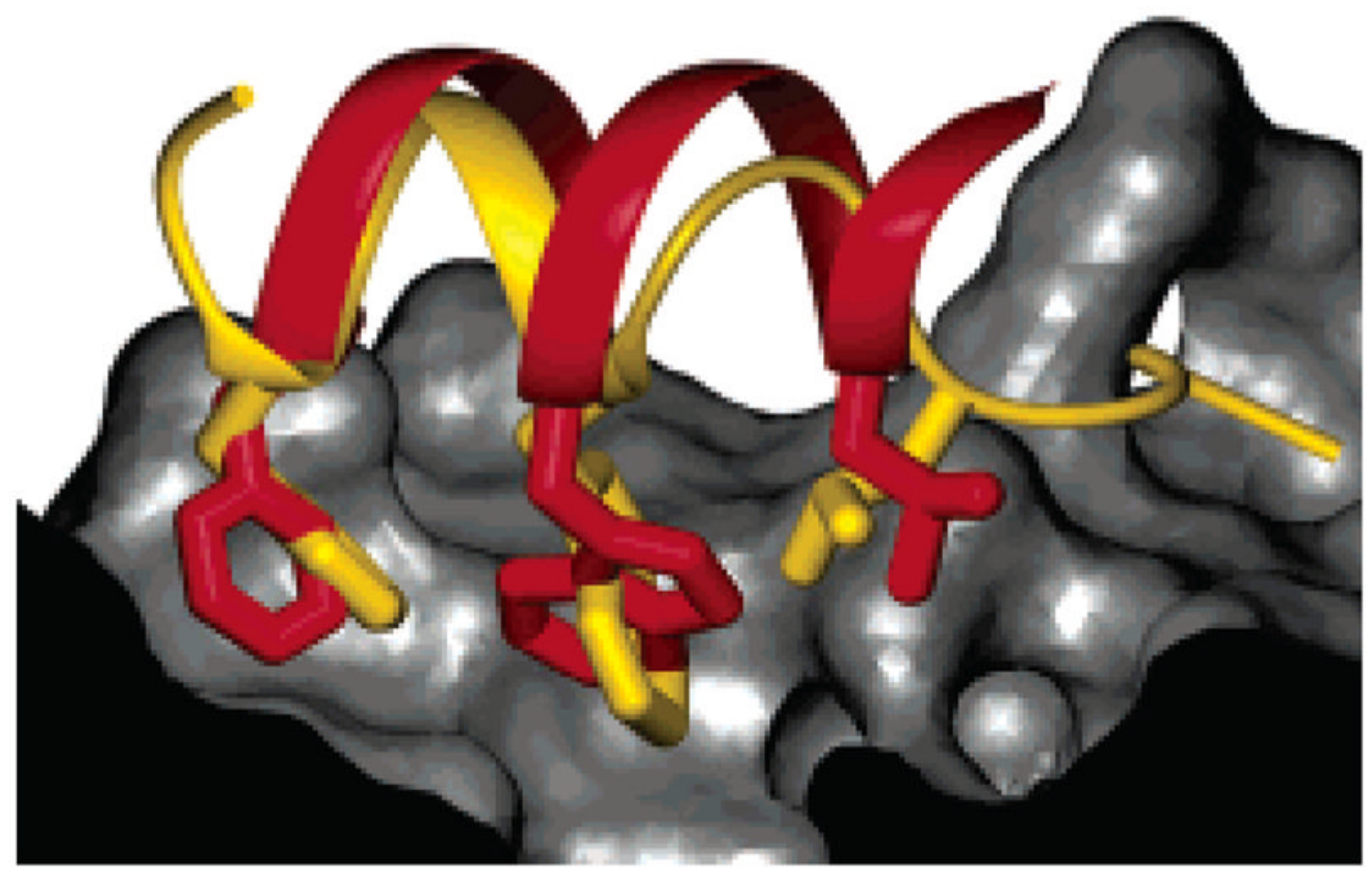

Figure 2.

Overlay of the methanol solution structure of $\boldsymbol{\beta 5 3}-\mathbf{1}$ (red ribbon and side chains) with the crystal structure of a p53AD-derived peptide (gold ribbon and side chains) bound to hDM2 (gray surface). ${ }^{23}$ Side chains of $\boldsymbol{\beta 5 3}-\mathbf{1}$ not implicated in recognition have been omitted, and part of the hDM2 surface has been cut away for clarity. 